\title{
DIMENSIONAMENTO HIDRÁULICO E AVALIAÇÃO DE UM SISTEMA DE IRRIGAÇÃO LOCALIZADA DE BAIXO CUSTO
}

Francicleiton Freires do Carmo ${ }^{1}$, Indalécio Dutra ${ }^{2}$, Adriana Alves Batista ${ }^{3}$, Rafael Oliveira Batista ${ }^{4}$, Mairton Gomes da Silva ${ }^{5}$

\section{RESUMO}

A ausência de tecnologia adequada é um dos maiores entraves à competitividade do agricultor familiar e uma barreira que dificulta sua sobrevivência no meio rural. Dito isto, objetivou-se com este trabalho o dimensionamento hidráulico e avaliação de desempenho do sistema de irrigação de baixo custo, bubbler, instalado no Assentamento Milagres em Apodi-RN. Após a implantação do referido sistema, avaliaram-se os indicadores de desempenho: coeficiente de uniformidade de Christiansen (CUC), coeficiente de uniformidade de distribuição (CUD), eficiência de aplicação (EA), coeficiente de variação da vazão (CVQ) e a vazão média unitária (Q). Utilizou-se o delineamento experimental inteiramente casualizado, onde os tratamentos consistiram de três períodos de avaliação (0, 84 e 168 horas de funcionamento), com cinco repetições. Os dados foram submetidos à análise de variância e quando significativo procedeu-se o teste de média. Todos os indicadores de desempenho avaliados apresentaram diferença estatisticamente significativa em função das horas de funcionamento. Os resultados indicaram que o CUC, CUD e EA decresceram; já o CVQ e a Q apresentaram resposta crescente com o aumento das horas de funcionamento. Mesmo decrescendo com o tempo de funcionamento, todos os indicadores de desempenho avaliados foram considerados aceitáveis, com exceção da EA após 168 horas de funcionamento. Com base nos resultados dos indicadores avaliados, pode-se concluir que o sistema de irrigação bubbler, foi bem dimensionado e operou dentro dos limites de desempenho aceitáveis.

Palavras-chaves: irrigação de baixo custo, sistema bubbler, uniformidade de distribuição de água

\section{ABSTRACT \\ HYDRAULIC DESIGN AND EVALUATION OF THE AN IRRIGATION SYSTEM LOCALIZED OF THE LOW COST}

The absence of appropriate technology is one of the biggest obstacles to the competitiveness of the family farmer and a barrier that makes it difficult to survive in rural areas. Therefore, the aim of this work the hydraulic design and performance evaluation of low-cost irrigation system, bubbler installed on Settlement Miracles in Apodi-RN. After the implantation of the system, we evaluated the performance indicators: coefficient of uniformity of Christiansen (CUC), distribution uniformity coefficient (CUD), application efficiency (EA), flow coefficient of variation of (CVQ) and average unit flow (Q). Utilized a completely randomized experimental design, where treatments consisted of three evaluation periods $(0,84$ and 168 hours of operation), with five repetitions. The data have been submitted to analysis of variance and when significant we proceeded to the average test. All performance indicators evaluated showed a statistically significant difference in terms of hours of operation. The results indicated that the CUC, CUD and EA decreased; already CVQ and Q showed increasing response with increasing hours of operation. Even reducing with the operating time, all performance indicators evaluated were considered acceptable, except EA after 168 hours of operation. Based on the results of this indicator, it can be concluded that the bubbler irrigation system has been well dimensioned and operated within acceptable performance limits.

Keywords: low cost irrigation, bubbler system, uniformity of water distribution

\section{Recebido para publicação em 02/06/2014. Aprovado em 02/03/2016.}

1 - Tecnólogo em Irrigação e Drenagem, Prefeitura Municipal de Iguatu, cleitontid@hotmail.com

2 - Engenheiro Agrônomo, Professor Adjunto da UFERSA/Mossoró - RN

3 - Tecnóloga em Irrigação e Drenagem, Mestre em Irrigação e Drenagem UFERSA/Mossoró - RN

4 - Engenheiro Agrícola, Professor Adjunto da UFERSA/Mossoró - RN

5 - Tecnólogo em Irrigação e Drenagem, Doutorando em Engenharia Agrícola na UFRB/Cruz das Almas - BA

302 REVENG

302-313p. ENGENHARIA NA AGRICUlTURA, VIÇOSA - MG, V.24 N.4, JULHO / AGOSTO 2016 


\section{INTRODUÇÃO}

A agricultura irrigada no Brasil tem sido praticada tanto por grandes como por pequenos produtores em projetos públicos de irrigação. Porém, em muitos casos, o que tem limitado o uso da irrigação por pequenos produtores são os custos iniciais de instalação dos sistemas de irrigação. Exemplos desses agricultores são os de assentamentos locados no semiárido do Nordeste brasileiro que muitas vezes são situados ás margens de rios ou lagoas, mas sem infraestrutura de irrigação (SILVA et al., 2011a).

Segundo Nascimento (2009a), devem-se desenvolver alternativas para difundir a irrigação para agricultura familiar, por proporcionar $\mathrm{o}$ aumento da produção, geração de emprego e renda e, consequentemente, diminuir o êxodo rural. Existem diversas tecnologias alternativas utilizadas na irrigação a custos mais baixos que podem propiciar a sua adoção por agricultores com pequeno poder financeiro, uma delas é o sistema bubber (COELHO et al., 2012a,b).

O sistema bubber difere dos outros tipos de irrigação localizada, pois funciona com baixa pressão, podendo usar apenas a gravidade como fonte de energia, que deve estar sob uma elevação de altura mínima de $1 \mathrm{~m}$, requer filtragem simples e pode operar sob pressões a partir de $10 \mathrm{kPa}$. Mesmo quando o bombeamento é necessário, bombas de baixa capacidade de elevação são suficientes (ANDRADE et al., 2002; SOUZA et al., 2005). Neste sistema, a água é lançada diretamente junto da planta em microbacias com frequência e vazões semelhantes ao sistema de microaspersão, mas de forma pontual, havendo um molhamento máximo de aproximadamente $67 \%$ da área. Devido à forma como o sistema bubbler aplica a água, ele fica restrito a culturas do tipo fruteiras ou árvores, não sendo indicado para pasto, grama ou culturas de espaçamento muito pequeno. As linhas principais, secundárias e laterais são enterradas no solo a profundidade que varia de 0,30 a $0,60 \mathrm{~m}$ (REYNOLDS et al., 1995).

O sistema bubber se apresenta muito bem ao pequeno produtor, pelo menor custo por hectare quando comparado com os demais sistemas de irrigação, pelo não requerimento de energia elétrica, a simplicidade de instalação, o manejo e a elevada eficiência de irrigação, estas características o credenciam como uma ferramenta importante no desenvolvimento da agricultura irrigada familiar.

$\mathrm{Na}$ literatura científica especializada, encontram-se publicados poucos trabalhos com o objetivo da difusão tecnológica deste sistema de irrigação, motivo pelo qual este trabalho tem maior relevância. Mas, pelos resultados já encontrados nesta literatura, evidencia-se que este sistema de irrigação é uma ferramenta com potencial para alavancar a produção agrícola advinda da agricultura familiar, sobretudo daquelas famílias rurais localizadas em regiões áridas e semiáridas caracterizada pelo baixo nível tecnológico dos agricultores familiares.

Diante da importância deste sistema de irrigação, contrastante à sua baixa disseminação, objetivou-se com o presente estudo, dimensionar e avaliar o desempenho do sistema de irrigação de baixo custo, bubbler, instalado em assentamento rural no semiárido do nordeste brasileiro.

\section{MATERIAL E MÉTODOS}

A presente pesquisa foi realizada no Assentamento rural Milagres em Apodi-RN, localizado a $\left(5^{\circ} 35^{\prime} 22^{\prime \prime} \mathrm{S}, 37^{\circ} 54^{\prime} 09^{\prime \prime}\right.$ W e altitude de $153 \mathrm{~m}$ do nível do mar). De acordo com a classificação de Köppen, o clima da região é do tipo 'BSw'h', tropical quente semiárido com temperatura média anual de $28,5^{\circ} \mathrm{C}$ e precipitação média anual de $772 \mathrm{~mm}$.

Foi dimensionado o sistema de irrigação bubbler com o auxílio do programa computacional Bubbler - versão1.1, desenvolvido pelo Departamento de Agricultura e Engenharia de Biossistemas da Universidade do Arizona. O programa foi alimentado com: o comprimento e largura da área, espaçamento da cultura, desnível longitudinal e transversal do terreno, diâmetros internos dos tubos comerciais, elevação e distância da fonte de água, profundidade de aterramento das tubulações e vazão unitária (mangueira).

O sistema de irrigação consistiu de uma linha principal de $25 \mathrm{~mm}$ de diâmetro, conectada à fonte de água, uma coluna com manômetro, quatro linhas laterais com diâmetro de $25 \mathrm{~mm}$ e 24 mangueiras emissoras de $5 \mathrm{~mm}$ de diâmetro conectadas às laterais (Figura 1). 


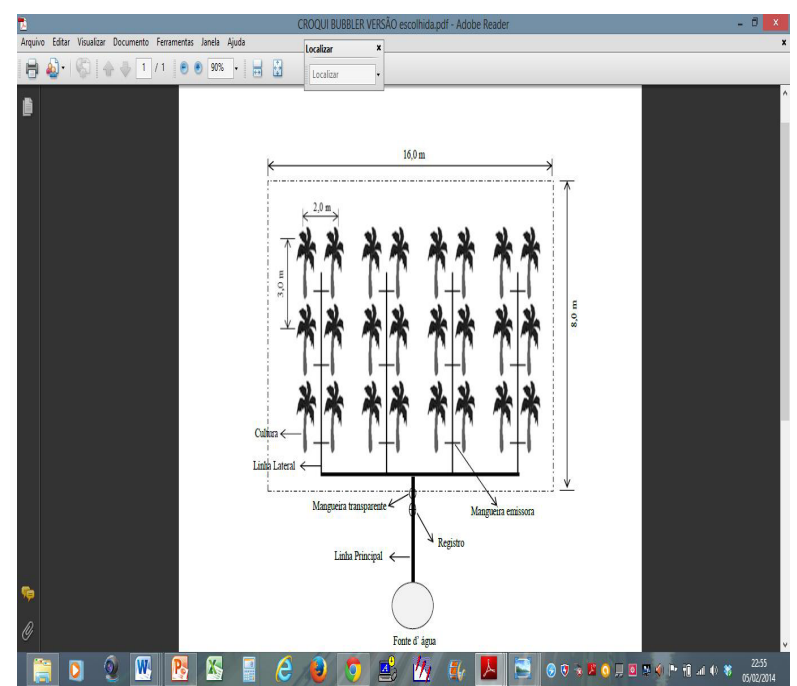

Fonte: Arquivo do primeiro autor.

Figura 1. Esquema do sistema de irrigação bubbler instalado na área (sem escala).
No mês de agosto de 2012, o sistema de irrigação bubbler foi instalado em uma área de 128 $\mathrm{m}^{2}(16 \mathrm{~m} \times 8 \mathrm{~m})$ para o cultivo de mamoeiro do grupo Formosa, híbrido Tainung, no espaçamento de $3 \times 2 \mathrm{~m}$. Esta cultura foi escolhida pelo potencial comercial na região e adaptação do sistema de irrigação ao sistema de cultivo.

A linha principal e as laterais foram enterradas a $0,30 \mathrm{~m}$ de profundidade, as mangueiras emissoras ficaram expostas, com alturas divididas em quatro grupos: 0,$64 ; 0,65 ; 0,67$ e $0,69 \mathrm{~m}$. Para fixar as alturas, utilizou-se um nível de mangueira, atribuindo a cota zero à superfície do solo próximo à coluna e manômetro. As mangueiras emissoras foram presas em ripas de madeira cravadas no solo, fixadas as alturas na saída da água estabelecida no dimensionamento. $\mathrm{O}$ suprimento de água foi realizado por um conjunto moto-bomba de 1,5 $\mathrm{CV}$ de potência e foi controlado por um registro
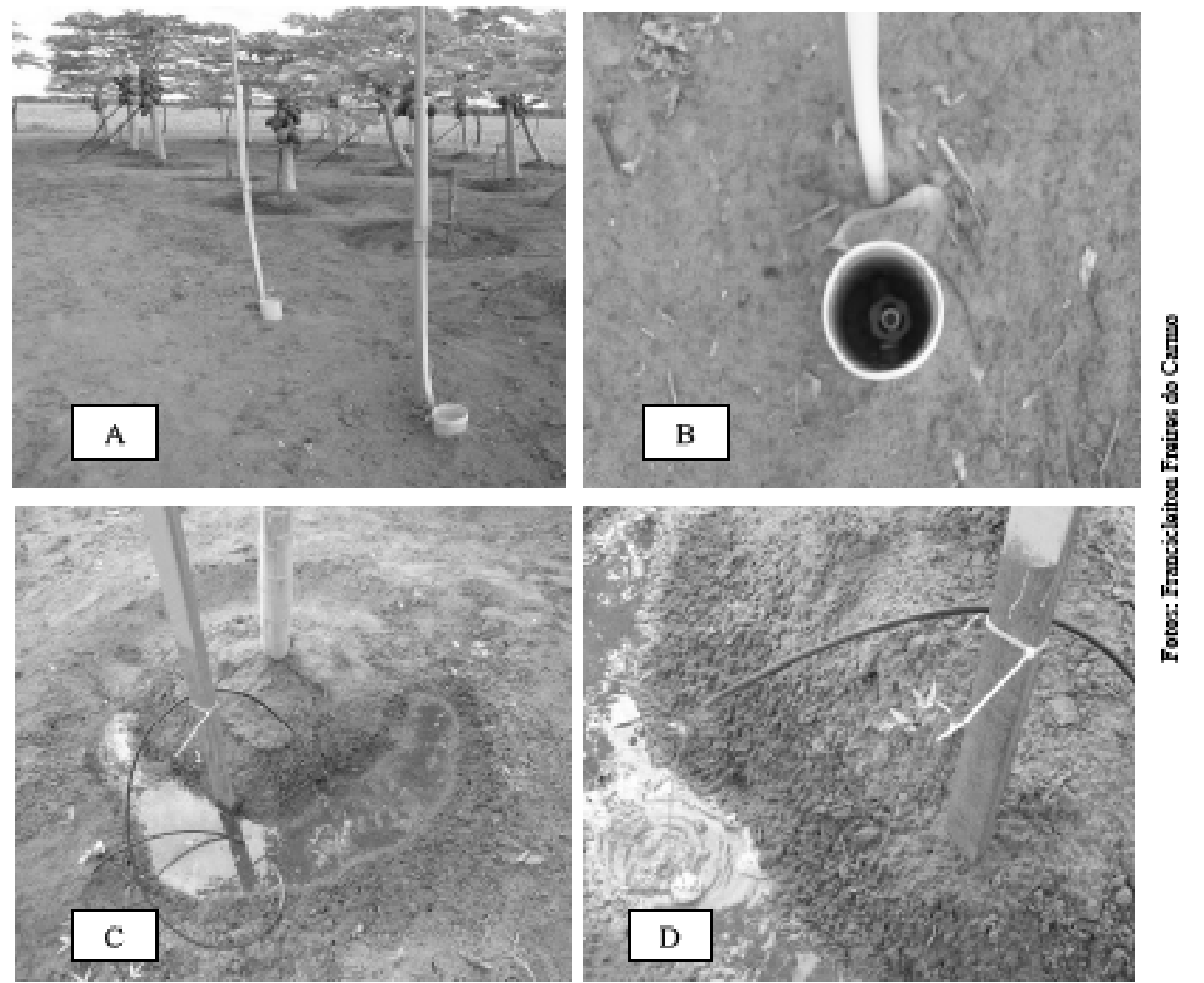

Figura 2. Detalhamento da coluna de controle da carga hidráulica - manômetro (A), registro para regulagem da vazão (B), mangueira emissora conduzindo água da lateral à microbacia (C) e saída de água da mangueira emissora e estaca de madeira como suporte desta.

\section{REVENG}


de gaveta localizado na coluna de controle, o qual permitia carga constante de $1,64 \mathrm{~m}$ de coluna de água, definido pelo aplicativo e marcado no manômetro (mangueira transparente de $16 \mathrm{~mm}$ de diâmetro) (Figura 2).

Foram realizadas três avaliações hidráulicas do sistema de irrigação bubbler, sendo: a primeira logo após a instalação (0 horas de funcionamento), a segunda e a terceira após 84 e 168 horas de funcionamento, respectivamente, em delineamento inteiramente casualizado com cinco repetições. Os tempos 84 e 168 horas, foram correspondentes de 4 e 8 meses de operação do sistema, respectivamente. $\mathrm{Na}$ avaliação hidráulica, determinou-se a vazão de cada uma das 24 mangueiras emissoras, com o auxílio de uma proveta de $500 \mathrm{~mL}$ graduada e cronômetro digital, e posteriormente os seguintes indicadores de desempenho hidráulico:

Coeficiente de Uniformidade de Christiansen (CUC), proposto por Christiansen (1942), adota o desvio médio como medida de dispersão (Equação 1).

$\mathrm{CUC}=\left[1-\frac{\sum_{\mathrm{i}=1}^{\mathrm{n}}\left|\mathrm{q}_{\mathrm{i}}-\mathrm{q}_{\mathrm{m}}\right|}{\mathrm{n} \times \mathrm{q}_{\mathrm{m}}}\right] \mathrm{x} 100$

em que,

CUC $=$ coeficiente de uniformidade de Christiansen, $\%$

$\mathrm{q}_{\mathrm{i}}=$ vazão do í-ésimo emissor, $\mathrm{L}$ h-1;

$\mathrm{q}_{\mathrm{m}}=$ vazão média de todos os emissores, $\mathrm{L}$ h-1; e

$\mathrm{n}=$ número de emissores amostrados.

Coeficiente de Uniformidade de Distribuição (CUD), proposto pelo Soil Conservation Service (1968), descrito na equação 2 .

$$
\text { CUD }=\left[\frac{\mathrm{q}_{25 \%}}{\mathrm{q}_{\mathrm{m}}}\right] \times 100
$$

em que,

CUD = coeficiente de uniformidade de distribuição, \%; $\mathrm{q}_{25 \%}=$ média das $25 \%$ menores vazões, $\mathrm{L}$ h- 1 ; e $\mathrm{q}_{\mathrm{m}}=$ vazão média de todos os emissores, L h-1.
Eficiência de aplicação (EA), recomendado por Vermeiren \& Jobling (1997) para sistema localizado. Este estabelece a relação entre o total de água aplicado e o total consumido pelas plantas (Equação 3).

$\mathrm{EA}=0,95 \times \mathrm{CUD}$

em que,

$\mathrm{EA}=$ eficiência de aplicação, \%; e

CUD $=$ coeficiente de uniformidade de distribuição, $\%$.

Coeficiente de variação de vazão (CVQ), expressa a relação do desvio padrão das vazões com a vazão média (Equação 4).

$\mathrm{CVQ}=\left[\frac{\mathrm{S}_{\mathrm{q}}}{\mathrm{q}_{\mathrm{m}}}\right] \times 100$

em que,

CVQ = coeficiente de variação de vazão, \%;

$\mathrm{Sq}=$ desvio padrão das vazões dos emissores amostrados, L h-1; e

$\mathrm{q}_{\mathrm{m}}=$ vazão média de todos os emissores, $\mathrm{L}$ h-1.

O desvio padrão foi determinado pela equação 5 abaixo.

$\mathrm{S}_{\mathrm{q}}=\left[\frac{\sum_{\mathrm{i}=1}^{\mathrm{n}}\left(\mathrm{q}_{\mathrm{i}}-\mathrm{q}_{\mathrm{m}}\right)}{\mathrm{n}}\right]^{0,5}$

A interpretação do CUC e CUD basearam-se na classificação apresentada por Mantovani (2001) (Quadro 1), a EA na de Bernardo et al. (2006) e o CVQ na ASAE (2003), (Quadro 2).

Os dados foram submetidos ao teste $\mathrm{F}$ da variância e, quando significativos, aplicou-se o teste de médias (Tukey) a 5\% de probabilidade. Os resultados estão apresentados em gráficos, para melhor visualização da tendência de comportamento dos indicadores de desempenho 
Quadro 1. Valores utilizados para classificação do CUC e CUD

\begin{tabular}{ccc}
\hline CUC (\%) & CUD (\%) & Classificação \\
\hline$>90$ & $>84$ & Excelente \\
$80-90$ & $68-84$ & Bom \\
$70-80$ & $52-68$ & Razoável \\
$60-70$ & $36-52$ & Ruim \\
$<60$ & $<36$ & Inaceitável \\
\hline
\end{tabular}

Fonte: Mantovani (2001)

Quadro 2. Valores utilizados para classificação da EA e CVQ

\begin{tabular}{cccc}
\hline EA (\%) & Classificação & CVQ (\%) & Classificação \\
\hline$\geq 95$ & Ideal & $\leq 10$ & Bom \\
$80-95$ & Aceitável & $10-20$ & Razoável \\
$<80$ & Inaceitável & $>20$ & Inaceitável \\
\hline
\end{tabular}

Fonte: Bernardo et al. (2006) e CVQ (ASAE, 2003)

ao longo dos períodos de avaliação com barra de erro padrão em torno da média, e em quadros, para facilitar a comparação dos valores e promover a respectiva classificação de acordo com as recomendações da literatura.

\section{RESULTADOS E DISCUSSÃO}

No quadro 3, encontram-se os resumos das análises de variância para o coeficiente de uniformidade de Christiansen (CUC), coeficiente de uniformidade de distribuição (CUD), eficiência de aplicação (EA), coeficiente de variação da vazão (CVQ) e vazão (Q). Nesta, é possível observar que todos os indicadores de desempenho avaliados sofreram efeito significativo pelo tempo de funcionamento do sistema de irrigação, mostrando que a qualidade da irrigação pode sofrer alteração ao longo da operação do sistema.

Para o CUC, verificou-se não haver diferença significativa entre as duas primeiras avaliações $(0 \mathrm{e}$ 84 horas de funcionamento), mas houve destas com a terceira avaliação (168 horas de funcionamento), conforme mostra figura 3 . O valor máximo $(91,61 \%)$ foi observado na primeira avaliação
(0 horas de funcionamento), já o valor mínimo $(86,86 \%)$ foi observado na terceira avaliação (168 horas de funcionamento), mostrando que houve uma redução de $5,17 \%$ entre os valores observados. Esta diferença pode ser considerada aceitável, levando-se em consideração o período de 8 meses em que o sistema foi avaliado no início (primeira) e no final (terceira) avaliação. Assim, por este indicador, a uniformidade de distribuição de água na área comportou-se muito bem ao longo dos períodos avaliados.

Na figura 3, é possível observar que os valores médios do CUC decresceram em função do tempo de funcionamento do sistema, no entanto, todos obtiveram classificação aceitável. Vermeiren e Jobling (1997) citam que os valores médios do CUC devem estar dentro da faixa de 85 a $95 \%$. Portanto, os valores obtidos nesta pesquisa foram superiores aos recomendados, expressando que o sistema apresentou boa uniformidade de distribuição de água.

Pela classificação de Mantovani (2001), o sistema apresentou desempenho considerado "Excelente" e "Bom", conforme quadro 4. Isto mostra a capacidade de manutenção da qualidade 
Quadro 3. Resumos das análises de variância para os indicadores estatísticos: CUC, CUD, EA, CVQ e Q obtidos na avaliação hidráulica do sistema de irrigação de baixo custo, bubbler

\begin{tabular}{ccccccc}
\hline \multirow{2}{*}{ Fonte de variação } & \multirow{2}{*}{ GL } & \multicolumn{5}{c}{ Quadrados Médios } \\
\cline { 3 - 7 } & & CUC & CUD & EA & CVQ & Q \\
\hline Horas de funcionamento & 2 & $33,5986^{*}$ & $84,1865^{* *}$ & $75,9703^{* *}$ & 58,2678 & $35,8658^{* *}$ \\
Erro & 12 & 4,9456 & 4,6714 & 4,2182 & 4,0325 & 0,02106 \\
\hline CV (\%) & & 2,48 & 2,61 & 2,61 & 14,94 & 0,50 \\
\hline
\end{tabular}

Nota: ${ }^{* *}$ Significativo ao nível de $1 \%,{ }^{*}$ significativo a $5 \%$ de probabilidade e ns - não significativo.

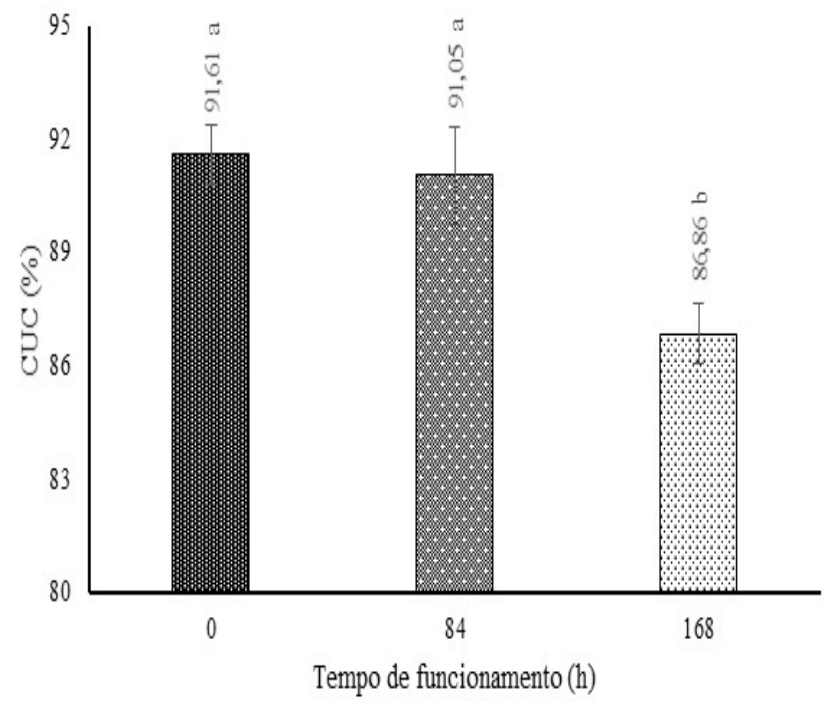

Nota: Letras diferentes diferem estatisticamente entre si, pelo teste de Tukey ao nível de 5\% de probabilidade.

Figura 3. Comportamento do CUC (\%), em função do tempo de funcionamento do sistema de irrigação bubbler.

da irrigação por este sistema ao longo de um grande período de operação, sem risco de comprometer a produção pela desuniformidade de distribuição da água.

Ainda segundo Mantovani (2001), o sistema que apresenta CUC superior a $88 \%$ pode ser utilizado para frutíferas ou até mesmo culturas de alto rendimento econômico, com sistema radicular pouco profundo. Isso mostra a adequacidade deste sistema para cultivos diversos, sendo mais indicado para aqueles de maior espaçamento entre as linhas de plantio.

O CUD apresentou resposta semelhante do CUC, ou seja, decresceu com o aumento nas horas de funcionamento do sistema. De acordo com a figura 4 abaixo, percebe-se que também não houve diferença estatística significativa entre as duas primeiras avaliações do sistema, havendo somente destas com a terceira avaliação. Comprovando a similaridade entre os indicadores de desempenho CUC e CUD.

Como mostra a figura 4, o CUD reduziu com aumento do tempo de funcionamento do sistema de irrigação, no entanto, todos os valores obtiveram classificação aceitável, sendo o valor máximo $(85,53 \%)$ obtido na primeira avaliação (0 horas de funcionamento) e o valor mínimo $(78,18 \%)$ obtido na terceira avaliação (168 horas de funcionamento), ocorrendo uma redução relativa $8,61 \%$ entre as duas avaliações. No estudo realizado por Cunha et al. (2013), em que estes avaliaram dois modelos de gotejadores ao longo de 100 e 200 horas 
Quadro 4. Valores médios do CUC nos diferentes tempos de funcionamento do sistema de irrigação bubbler e sua respectiva classificação

\begin{tabular}{ccc}
\hline Tempo de funcionamento $(\mathrm{h})$ & Valor médio do CUC $(\%)$ & Classificação \\
\hline 0 & 91,60 & Excelente \\
84 & 91,05 & Excelente \\
168 & 86,86 & Bom \\
\hline
\end{tabular}

Fonte: Dados da pesquisa.

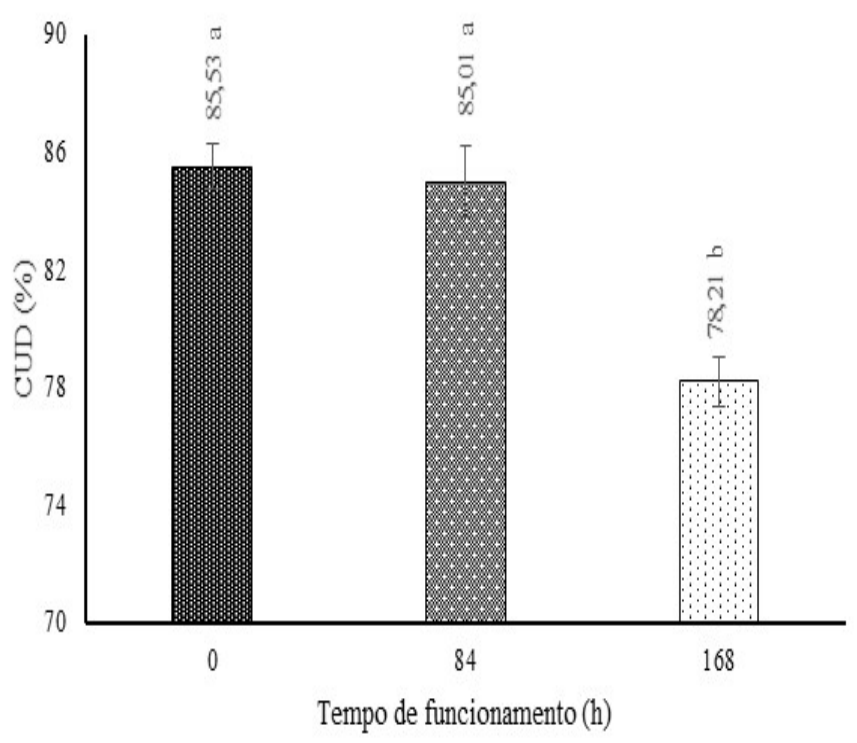

Nota: Letras diferentes diferem estatisticamente entre si, pelo teste de Tukey ao nível de 5\% de probabilidade.

Figura 4. Comportamento do CUD (\%), em função do tempo de funcionamento do sistema de irrigação bubbler.

funcionamento, foi possível observar um aumento de $2,52 \%$ e uma redução de $7,4 \%$, respectivamente, entre as 100 e $200 \mathrm{~h}$. Isso mostra que a partir de 100 horas de funcionamento pode haver uma tendência de redução da uniformidade de distribuição de água na área.

De acordo com o quadro 5, observando a classificação apresentada por Mantovani (2001), os valores do CUD classificaram-se como "Excelente" (0 e 84 horas de funcionamento) e "Bom" (168 horas de funcionamento). Isso mostra que mesmo após um período longo de funcionamento do sistema ( 8 meses entre a primeira e terceira avaliação), a uniformidade de distribuição de água na área foi mantida, fator muito importante principalmente para cultivos sensíveis ao estresse hídrico. Segundo López et al. (1992), na irrigação localizada o CUD é o indicador mais utilizado na avaliação de desempenho, pois este possibilita uma medida mais rigorosa, dando maior peso às plantas que recebem menos água.

Em estudos conduzidos por Dutra (2002), Souza et al. (2005) e Arraes et al. (2007), ao avaliarem o desempenho do sistema bubbler, encontraram valores do CUC de 93,12; 96,64 e 93,70\%, e CUD de 89,92; 95,85 e 92,0\%, respectivamente, comprovando o bom desempenho deste sistema. Estes resultados são semelhantes aos encontrados neste trabalho, o que mostra a possibilidade de acerto no dimensionamento deste sistema de irrigação, que é o principal componente para que o seu bom desempenho hidráulico em campo. Rodrigues et al. (2013), avaliando um sistema de irrigação por gotejamento, encontraram CUC 
Quadro 5. Valores médios do CUD nos diferentes tempos de funcionamento do sistema de irrigação bubbler e sua respectiva classificação

\begin{tabular}{ccc}
\hline Tempo de funcionamento $(\mathrm{h})$ & Valor médio do CUD, em $\%$ & Classificação \\
\hline 0 & 85,53 & Excelente \\
84 & 85,01 & Excelente \\
168 & 78,18 & Bom \\
\hline
\end{tabular}

Fonte: Dados da pesquisa

de $90,4 \%$ e CUD de $83,31 \%$, valores inferiores a alguns encontrados neste trabalho, mostrando que este sistema pode, desde que bem dimensionado e operado, ter o desempenho comparado ao gotejamento.

O indicador estatístico Eficiência de Aplicação (EA) apresentou o mesmo comportamento do CUD, pois foi obtido pelo produto entre o CUD e o coeficiente de transmissividade (Ks), que no presente adotou-se em $95 \%$. A EA é um parâmetro definido pela relação entre a quantidade de água incorporada ao solo, até a profundidade efetiva do sistema radicular da cultura e a quantidade de água retirada da fonte hídrica (BENİCIO et al., 2009). Segundo Souza (2001), é um parâmetro muito usado no dimensionamento e manejo de sistemas de irrigação, pois indica a capacidade funcional e operacional do sistema naquelas condições.

De acordo com a figura 5, observa-se que a EA apresentou comportamento decrescente com o aumento do tempo de funcionamento do sistema de irrigação bubbler. Assim como no CUC e CUD, também não houve diferença estatística significativa entre as duas primeiras avaliações $(0 \mathrm{e}$ 84 horas de funcionamento), mas houve destas com a terceira avaliação (168 horas de funcionamento), sendo o valor máximo $(81,25 \%)$ obtido na primeira avaliação ( 0 horas de funcionamento) e o valor mínimo (74,27\%) obtido na terceira avaliação (168 horas de funcionamento), ocorrendo uma redução relativa $8,59 \%$ entre estas duas avaliações.

Isso mostra a correlação entre os indicadores CUC, CUD e EA, e indica que há uma tendência de piora na uniformidade de distribuição e eficiência do uso da água no sistema de irrigação bubbler, com o aumento tempo de funcionamento. As causas para isto são variadas, uma vez que diversos fatores podem interferir isolados ou coletivamente no desempenho deste sistema de irrigação, entre as principais encontram-se a obstrução das mangueiras emissoras, a oscilação de pressão da rede e falhas hidráulicas no dimensionamento e operação.

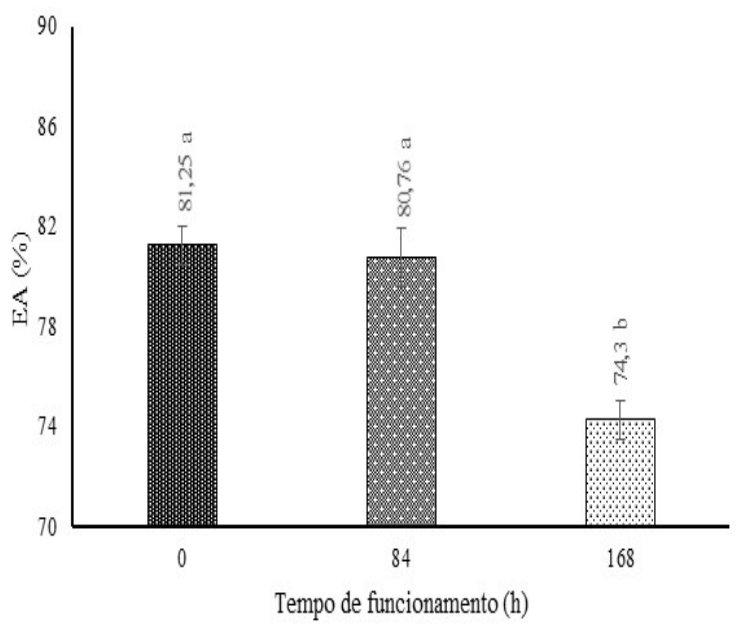

Nota: Letras diferentes diferem estatisticamente entre si, pelo teste de Tukey ao nível de 5\% de probabilidade.

Figura 5. Comportamento da EA (\%), em função do tempo de funcionamento do sistema de irrigação bubbler.

Utilizando a metodologia proposta por Bernardo et al. (2006), os valores da EA foram classificados em "Aceitável" nas duas primeiras avaliações (0 e 84 horas de funcionamento) e "Inaceitável" na terceira (168 horas de funcionamento), conforme mostra o quadro 6.

No momento em que a EA é inaceitável, certamente, houve falhas durante o manejo e operação do sistema de irrigação, se não foi no 
Quadro 6. Valores médios da EA nos diferentes tempos de funcionamento do sistema de irrigação bubbler e sua respectiva classificação

\begin{tabular}{ccc}
\hline Tempo de funcionamento $(\mathrm{h})$ & Valores médios da EA $(\%)$ & Classificação \\
\hline 0 & 81,25 & Aceitável \\
84 & 80,76 & Aceitável \\
168 & 74,27 & Inaceitável \\
\hline
\end{tabular}

Fonte: Dados da pesquisa.

dimensionamento. Para que isto não ocorra, ações preventivas devem ser adotas, como limpeza periódica do sistema de filtragem e da rede de tubulações e mangueiras emissoras, verificação de furos nas mangueiras, avaliação diária da pressão e com certa frequência a avaliação do sistema de irrigação.

O CVQ mostrado na figura 6 apresentou comportamento inverso ao CUC, CUD e EA, ou seja, sua resposta ao longo do tempo foi crescente. Não houve diferença estatística significativa entre as duas primeiras avaliações ( 0 e 84 horas de funcionamento), mas houve destas com a terceira avaliação (168 horas de funcionamento). $\mathrm{O}$ valor máximo do CVQ (17,3\%) foi observado na terceira avaliação (168 horas de funcionamento) e o menor $(10,82 \%)$ foi observado na primeira avaliação (0 horas de funcionamento), um aumento no valor do CVQ em $37,46 \%$, ao longo do tempo de funcionamento do sistema de irrigação.

O aumento do CVQ ao longo do tempo pode estar relacionado a possíveis obstruções nas mangueiras emissoras, provocadas por insetos ou outras sujeiras que possam ficar acumuladas, às características de fabricação do emissor (mangueiras emissoras) de possuírem alta variabilidade na qualidade de fabricação, principalmente pela despadronização dos diâmetros internos, e ainda à ocorrência de oscilação de pressão no momento da avaliação, porque, uma vez que o sistema é de baixa pressão, qualquer oscilação provoca alta variação de vazão. Esse mesmo tipo de comportamento foi verificado por Cunha et al. (2013) na avaliação de sistema de irrigação por gotejamento, ou seja, os valores da uniformidade de distribuição reduziram ao longo do período de operação, enquanto que os valores de coeficiente de variação de vazão aumentaram.

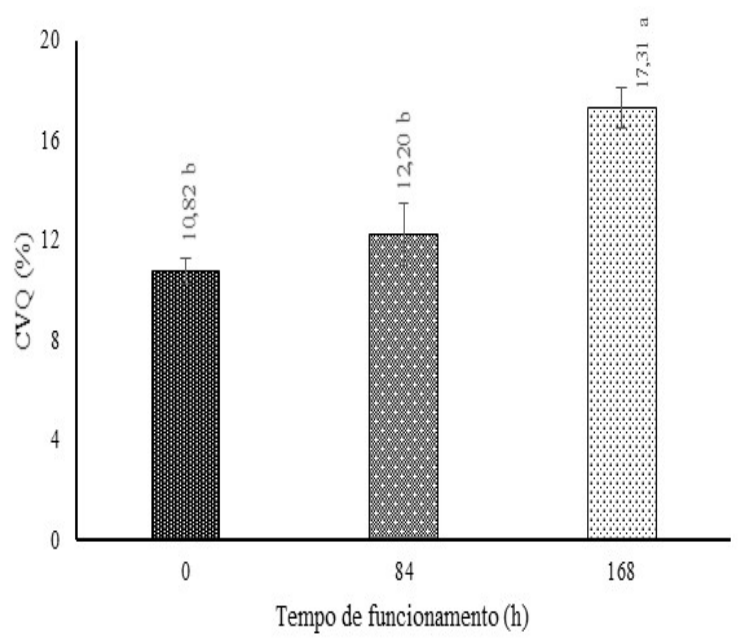

Nota: Letras diferentes diferem estatisticamente entre si, pelo teste de Tukey ao nível de 5\% de probabilidade.

Figura 6. Comportamento do CVQ (\%), em função do tempo de funcionamento do sistema de irrigação bubber.

Segundo a ASAE (2003), todos os valores do CVQ foram classificados como "Razoável" conforme mostra quadro 7. Esse comportamento já era esperado, visto que ao longo do tempo a vazão tende a se tornar desuniforme, pelos motivos anteriormente mencionados, que provocam consequentemente aumento do CVQ. Por isso, são de suma importância um acompanhamento e avaliação periódica do sistema de irrigação, para identificação e correção de problemas que possam afetar a uniformidade de distribuição da vazão na área.

$\mathrm{Na}$ figura 7, observa-se que para a vazão (Q) houve diferença estatística significativa entre as três avaliações $(0,84$ e 168 horas de funcionamento). O valor máximo médio da $\mathrm{Q}$ ( 31 , $\left.15 \mathrm{~L} \mathrm{~h}^{-1}\right)$ foi observado na segunda avaliação (84

\section{REVENG


Quadro 7. Valores médios do CVQ nos diferentes tempos de funcionamento do sistema de irrigação bubbler e sua respectiva classificação

\begin{tabular}{ccc}
\hline Tempo de funcionamento $(\mathrm{h})$ & Valores médios do CVQ $(\%)$ & Classificação \\
\hline 0 & 10,82 & Razoável \\
84 & 12,20 & Razoável \\
168 & 17,30 & Razoável \\
\hline
\end{tabular}

Fonte: Dados da pesquisa.

horas de funcionamento) e o menor $\left(25,85 \mathrm{~L} \mathrm{~h}^{-1}\right)$ foi observado na terceira avaliação (168 horas de funcionamento), uma redução de $17,01 \%$. Era esperada uma maior vazão na primeira avaliação, porém pode ter acontecido no momento da coleta uma das possíveis causas de variação de vazão, como a oscilação de pressão, ou obstruções na rede e mangueiras emissoras oriundas da montagem do sistema.

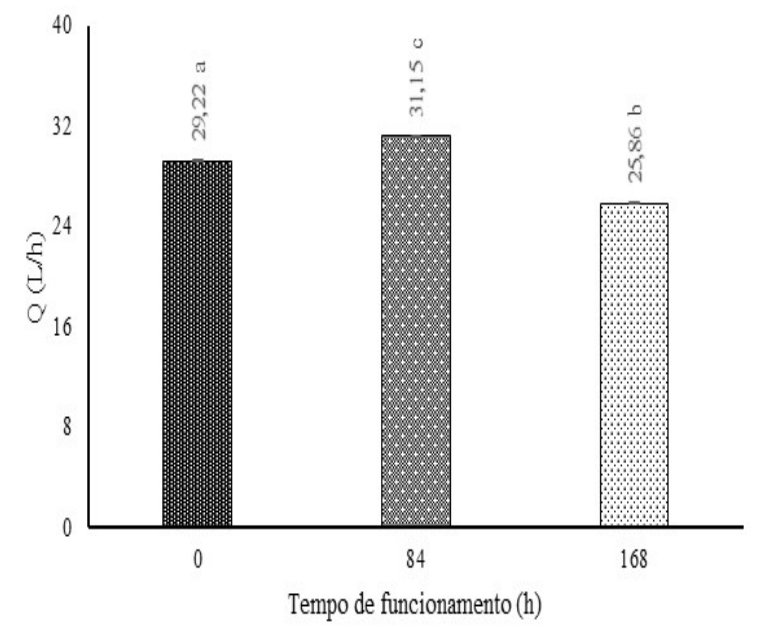

Nota: Letras diferentes diferem estatisticamente entre si, pelo teste de Tukey ao nível de 5\% de probabilidade.

Figura 7. Comportamento da $\mathrm{Q}\left(\mathrm{L} \mathrm{h}^{-1}\right)$, em função do tempo de funcionamento do sistema de irrigação bubber.

Essa variação de vazão interfere diretamente para um desempenho irregular do sistema de irrigação e isto pode ser fruto da falta ou insuficiente manutenção no sistema, uma vez que as linhas laterais só foram abertas antes das avaliações do sistema. As causas encontradas foram algumas mangueiras emissoras danificadas por animais silvestres e outras obstruídas por insetos e imprecisão nas alturas das mangueiras emissoras na recolocação destas, quando se soltavam da estaca, pois não voltada à mesma altura inicial determinada pelo software.

Outra possível causa pode ser a variação do diâmetro interno dos tubos usados como emissores, uma vez que no processo de fabricação não se consegue uniformidade elevada neste tipo de material. Uma solução para este problema pode ser o uso de outros emissores alternativos de baixo custo, ou até mesmo comerciais como fez Pachico e Levien (2014).

A melhoria da uniformidade de distribuição de água na área pode ser obtida por meio da adoção de práticas simples de manejo, como limpeza periódica mais criteriosa do sistema de filtragem, possibilitando maior pressão nos pontos de emissão, assim como desentupimento dos emissores e limpeza das linhas laterais.

$\mathrm{Na}$ avaliação de quatro sistemas de irrigação alternativos, Silva et al. (2011b) obtiveram valores de vazão média $\left(\mathrm{Q}\right.$, em $\left.\mathrm{L} \mathrm{h}^{-1}\right)$, coeficiente de variação $(\mathrm{CV}$, em \%) e eficiência de uniformidade (EU, em \%) de 78,8; 21,1 e 78,7 (no sistema de microaspersão artesanal); 17,8; 26,3 e 66,8 (gotejamento artesanal); 45,1; 66,0 e 34,3 (no sistema xique-xique com conector de $4 \mathrm{~mm}$ de diâmetro em cada furo da mangueira) e 182,9; 44,2 e 51,8 (no sistema bubbler), respectivamente.

Ainda de acordo com Silva et al. (2011b), o sistema bubbler apresentou valores de CV elevado e EU baixo, indicando ter sido um método de baixa uniformidade de distribuição de água e que a razão para o baixo desempenho desses sistemas, comparado aos sistemas comerciais, foi o fato de que, por serem sistemas idealizados para pequena agricultura, com propósito de baixo custo, os emissores no caso do gotejamento, da microaspersão foram construídos artesanalmente, 
portanto com considerável variabilidade nas dimensões dos orifícios de saída de água, o que era esperado. No entanto, outras causas também podem ter interferido, como falhas hidráulicas de dimensionamento, operação e manutenção.

Para o sistema bubbler, pode observar que os resultados obtidos neste trabalho foram melhores, ou seja, os indicadores de desempenho CV foram menores, com consequente maior uniformidade de distribuição de água na área, e ainda a EU que pode ser comparado com o CUC ou CUD também apresentou valores inferiores, inferindo que, quando o sistema é bem dimensionado e operado, pode apresentar desempenho satisfatório.

\section{CONCLUSÕES}

- Todos os indicadores de desempenho avaliados mostraram diferença significativa em função do tempo de funcionamento do sistema de irrigação bubbler;

- Os valores do CUC, CUD e da EA decresceram com o aumento do tempo de funcionamento do sistema, ao contrário do CVQ e da vazão;

- Os indicadores de desempenho mostraram que o sistema de irrigação bubbler, apresentou bom desempenho em campo, demonstrando que foi hidraulicamente bem dimensionado.

\section{AGRADECIMENTOS}

Ao $\mathrm{CNPq}$ pelo apoio financeiro do projeto, a CAPES pela bolsa de pós-graduação ao primeiro e terceiro autor e a UFERSA pelo transporte e infraestrutura disponibilizados.

\section{REFERÊNCIAS BIBLIOGRÁFICAS}

AMERICAN SOCIETY OF AGRICULTURAL AND BIOLOGICAL ENGINEERS-ASABE EP 405. Design and installation of microirrigation systems. ASAE Standards, St. Joseph, p.900-905, 2003.

ANDRADE, E.M.; SOUZA, I.H.; SILVA, E.L. Análise dos custos fixos de um sistema de irrigação localizado por gravidade-bubbler. Revista Ciência Agronômica, Fortaleza, v.33, n.1, p.64-69, 2002.

ARRAES, F.D.D.; ANDRADE, E.M.; SOUZA, I.H.; COSTA, M.P.; BRITO, J.L.D.; MIRANDA, C.A.; PALÁCIO, H.Q.A. Análise da uniformidade de aplicação de água em sistema de irrigação de baixa pressão, bubbler. In: Congresso Nacional de Irrigação e Drenagem, 17, 2007, Mossoró. Anais... ABID, 2007, p.23-29.

BENÍCIO, F.R.; CARVALHO, C.M.; ELOI, W.M.; GONÇALVES, F.M.; BORGES, F.R.M. Desempenho de um sistema de irrigação por microaspersão na cultura da goiaba em BarbalhaCE. Revista Brasileira de Agricultura Irrigada, Fortaleza, v.3, n.2, p.55-61, 2009.

BERNARDO, S.; SOARES, A.A.; MANTOVANI, E.C. Manual de irrigação. 8. ed. Viçosa: UFV, 2006. 625p.

CHRISTIANSEN, J.E. Irrigation by sprinkling. Berkeley, University of California: Agricultural Experiment Station, 1942. 124p. (Bulletin, 670).

COELHO, E.F.; SILVA, T.S.M.; PARIZOTTO, I.; SILVA, A.J.P.; SANTOS, D.B. Sistemas de irrigação de baixo custo para agricultura familiar. Cruz das Almas, BA. Embrapa Mandioca e Fruticultura, 2012a. 8p. (Circular Técnica, 106).

COELHO, E.F.; SILVA, T.S.M.; SILVA, A.J.P.; PARIZOTTO, I.; CONCEIÇÃO, B.S.; SANTOS, D.B. Sistemas de irrigação de baixo custo para agricultura familiar de assentamentos ribeirinhos do semiárido. In: GHEYI, H.R.; PAZ, V.P.S.; MEDEIROS, S.S.; GALVÃO, C.O (eds.). Recursos hídricos em regiões semiáridas: Estudos e aplicações. - Campina Grande, PB: Instituto Nacional do Semiárido - INSA, Cruz das Almas, BA: Universidade Federal do Recôncavo da Bahia - UFRB, 2012b, cap.5, p.100-115.

CUNHA, F.N.; OLIVEIRA, R.C.; SILVA, N.F.; MOURA, L.M.F.; TEIXEIRA, M.B.; FILHO, R.R.G. Variabilidade temporal da uniformidade de distribuição em sistema de gotejamento. Revista

\section{REVENG}


Brasileira de Agricultura Irrigada, Fortaleza, v.7, n.4, p.248-257, 2013.

DUTRA, I. Distribuição da água e avaliação da salinidade no solo em cultivo de goiabeira irrigado por sistema localizado de baixa pressão. 2002. 73p. Dissertação (Mestrado em Irrigação e Drenagem) - Universidade Federal do Ceará, Fortaleza, 2002.

LÓPEZ, J.R.; ABREU, J.M.H.; REGALADO, A.P.; HERNÁNDEZ, J.F.G. Riego Localizado. Madrid, Mundi - Prensa, 1992. 405p.

MANTOVANI, E.C. AVALIA: Programa de avaliação da irrigação por aspersão e localizada. Viçosa: UFV, 2001.

MERRIAM, J.L.; KELLER, J. Farm Irrigation System Evaluation: A Guide for Management. Logan: Utah State University, 1978. 271p.

NASCIMENTO, A.K.S.; SOUZA, R.O.R.M.; LIMA, S.C.R.V.; CARVALHO, C.M.; ROCHA, B.M.; LEITE, K.N. Desempenho hidráulico e manejo da irrigação em sistema irrigado por microaspersão. Revista Brasileira de Agricultura Irrigada, Fortaleza, v.3, n.1, p.39-45, 2009 b.

NASCIMENTO, J.M.S.; LIMA, L.A.; CARARO, D.C.; CASTRO, E.M.; SILVA, M.V.G. Avaliação da uniformidade de aplicação de água em um sistema de gotejamento para pequenas propriedades. Ciência e Agrotecnologia, Lavras, v.33, Edição Especial, p.1728-1733, 2009a.

PACHICO, I.W.L.; LEVIEN, S.L.A.. Avaliação de emissores de baixo custo de irrigação localizada submetidos à baixa pressão. Irriga, Botucatu, $1^{\text {a }}$ Edição Especial, p.55-61, 2014.

REIS, E.F.; BARROS, F.M.; CAMPANHARO, M.; PEZZOPANE, J.E.M. Avaliação do desempenho de sistemas de irrigação por gotejamento. Engenharia na Agricultura, Viçosa, v.13, n.2, p.74-81, 2005.

REYNOLDS, C.A. Design and evaluation of bubbler irrigation systems. 1993. 134f. Master's Thesis (Biosystem Agricultural Engineering)-
University of Arizona, Arizona, 1993.

REYNOLDS, C.A.; YITAYEW, M.; PETERSEN, M. Low-head bubbler irrigation systems. Part I: Design. Agricultural Water Management, Amsterdam, v.29, n.1, p.1-24, 1995.

RODRIGUES, R.R.; COLA, M.P.A.; NAZÁRIO, A.A.; AZEVEDO, J.M.G.; REIS, E.F. Eficiência e uniformidade de um sistema de irrigação por gotejamento na cultura do cafeeiro. Ambiência, Guarapuava, v.9, n.2, p.323-334, 2013.

SILVA, A.J.P.; SILVA, V.P.; SÁ, T.; COELHO, E.F.; CARVALHO, A.J.A. Crescimento e produtividade de alface irrigada por diferentes sistemas de irrigação de baixo custo utilizando captação de água da chuva. In: Congresso Nacional de Irrigação e Drenagem, 21, 2011a, Petrolina. Anais... ABID, 2011. CD Rom.

SILVA, T.S.M.; COELHO, E.F.; CONCEIÇÃO, B.S.; SILVA, A.J.P. Sistemas de irrigação para agricultura familiar em assentamento do semiárido baiano. In: Congresso Brasileiro de Engenharia Agrícola, 40, 2011b, Cuiabá-MT. Anais... SBEA, 2011. CD Rom.

SOIL CONSERVATION SERVICE. National Engineering Handbook. Washington: Sprinkler Irrigation, 1968. Section 15, Chapter 11. 83p.

SOUZA, I.H.; ANDRADE, E.M.; SILVA, E.L. Avaliação hidráulica de um sistema de irrigação localizada de baixa pressão, projetado pelo software "Bubbler". Engenharia Agrícola, Jaboticabal, v.25, n.1, p.264-271, 2005.

SOUZA, R.O.R.M. Desenvolvimento e avaliação de um sistema de irrigação automatizada para áreas experimentais. 2001. 70f. Dissertação (Mestrado em Agronomia) - Escola Superior de Agricultura "Luiz de Queiroz", Universidade de São Paulo, Piracicaba, 2001.

VERMEIREN, L.; JOBLING, G.A. Irrigação localizada. Campina Grande: UFPB, 1997. 184p. (Estudos FAO - Irrigação e Drenagem, 36). 